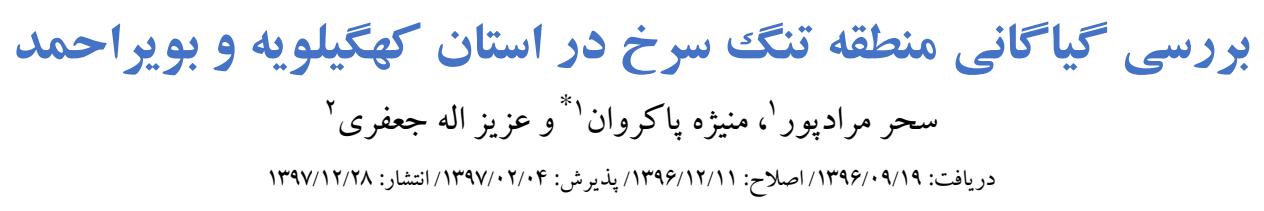

$$
\begin{aligned}
& \text { 'دانشكده علوم زيستى دانشگاه الزهرا ، تهران، ايران } \\
& \text { 'دانشكده علوم، دانشگاه ياسوج، ياسوج، ايران } \\
& \text { "مسئول مكاتبات: pakravan@alzahra.ac.ir" }
\end{aligned}
$$

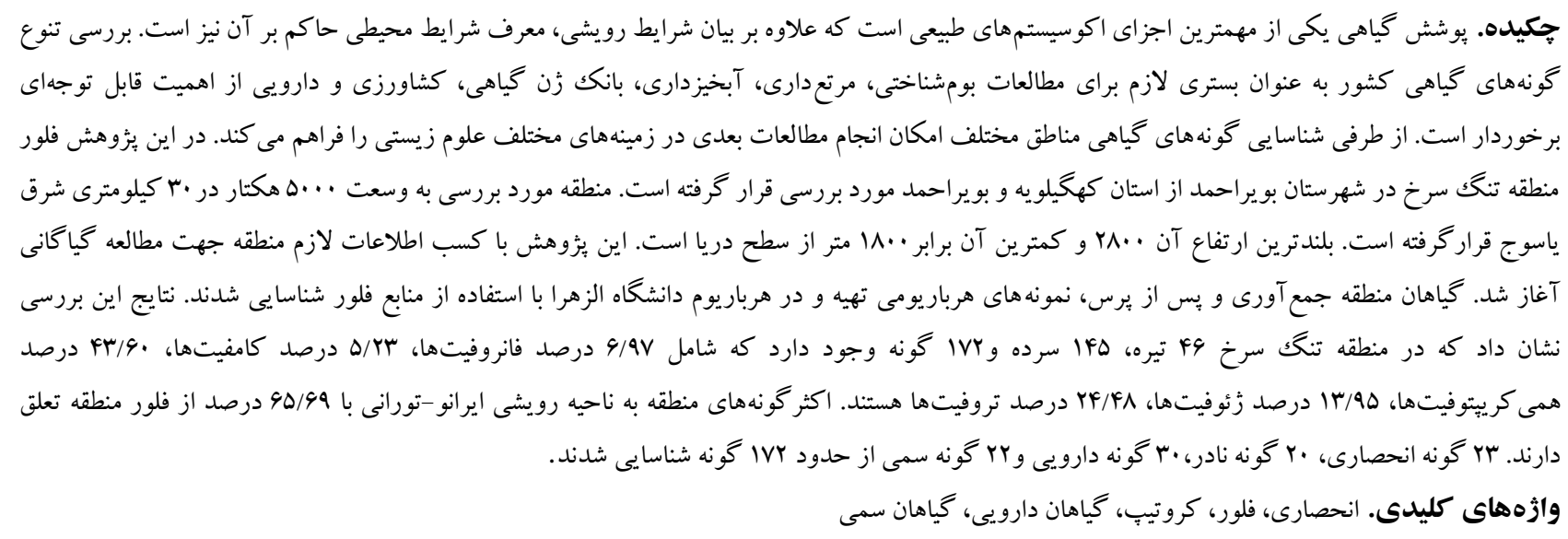

\title{
The Floristic study of Tang-sorkh region of Kohgiloyeh va Boyerahmad province
}

Sahar Moradpoor ${ }^{1}$, Maneezheh Pakravan ${ }^{1 *} \&$ Azizollah Jafari ${ }^{2}$

Received 10.12.2017/ Revised 02.03.2018/ Accepted 11.03.2018/ Published 19.03.2019

${ }^{1}$ Faculty of Biological Sciences, Alzahra University, Tehran, Iran

${ }^{2}$ Faculty of Biological Science, Yasuj University, Yasuj, Iran

*Correspondent author: pakravan@alzahra.ac.ir

\begin{abstract}
Vegetation is one of the most important parts of natural ecosystems that in addition to express of the growth conditions, it also shows the influence of ecological factors on the environmental condition. Investigations of plant diversity of a country are necessary for studyingthe ecosystem, pasture, plant gene bank, agriculture and medicine. On the one hand, identification of plant species of different regions provide feasibility of doing of next studies in the different fields of biological science. In this research, the flora of Tang sorkh region in the Kohgiloyeh county from Kohgiloyeh va boyerahmad province has been investigated. The area of mentioned region is 5000 hectares and it is located in 30 kilometers from the east of Yasuj. Maximum altitude of the area was between 1800-2800 m. Our research has started by collecting the essential information of the region. The plants of region have been collected and prepared for studying. Several floras were used for identification the specimens and they are preserved in Alzahra University herbarium (ALUH). This investigation showed that in the Tang sorkh region, there are 46 families, 145 genera, 172 species that include 6/97\% of phanerophytes, 5/23\% chamephyts, $43 / 60 \%$ hemicryptophytes, $13 / 95 \%$ geophytes, $28 / 48 \%$ therophytes. Analyses shows that most of species of region belongs to the Iran-o-Turanian region which contanins $5 / 69 \%$ of region's flora. 23 species are endemic to Iran, 20 of which are rare species, 30 species are medicinal plant, 22 of which are poison. Totaly about 172 species has identified.
\end{abstract}

Keywords. Chorotype, endemic, flora, medicinal plant, poisonous plants 
هاى شمال و شمال غرب شهرستان جرام (اشخر و بهن) گونههاى

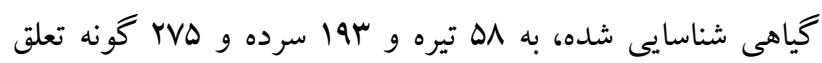

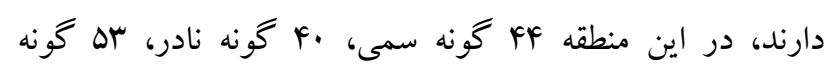

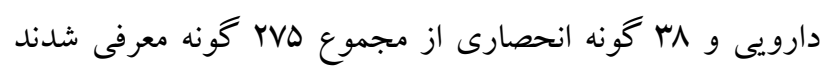
Y Yه (Janipour, 2015) Fونه وجود دارد كه در اين منطقه FV FV Fونه انحصارى ايران،

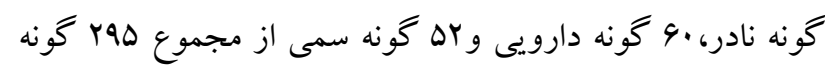
معرفى شدند (Zarifian, 2014).

منطقة تحت مطالعه منطقه تنك سرخ واقع در ·ـ كيلومترى شرق ياسوج در استان

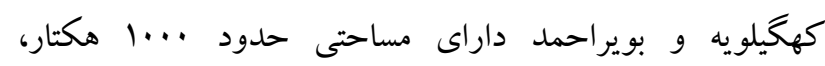

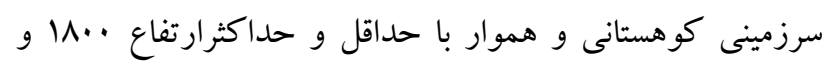

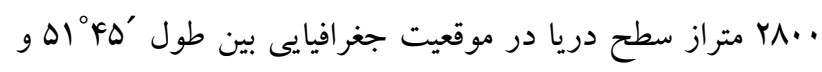

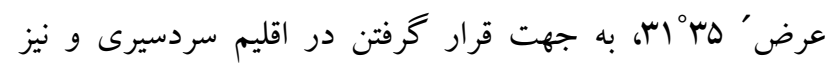

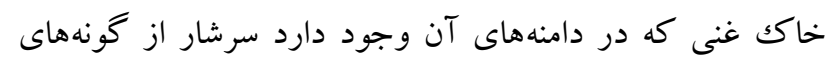
كياهى متنوع و حتى منحصر به فرد است. ميانگين بارش ساليانه حدود كY4 ميلىمتر بر آورد شده است كه داراى توزيع سالانه تقريباً مناسبى است. ميانگين دماى ساليانه در مردادماه با ميا ميانگين

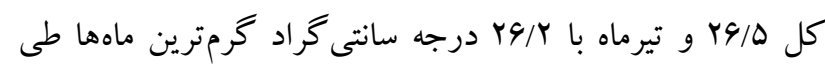
آمار TV

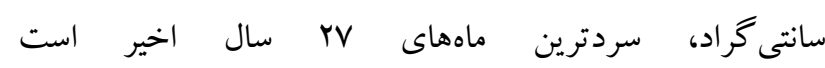
Meteorological Organization Kohgiluyeh and )

.(Boyer-ahmad province

\section{مواد و روشها}

جهت بررسىهاى اوليه نقشه جغرافيايى درمقياس

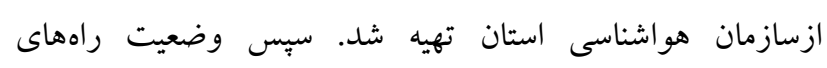
ارتباطى، يوشش گياهى و مناطق مسكونى مورد مطالعه قرار گرفت.

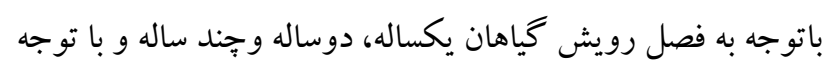

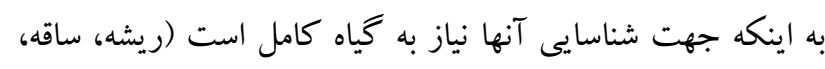

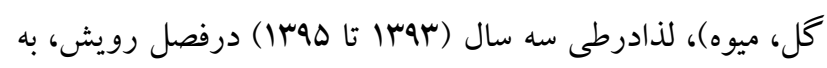
طورمرتب گياهان منطقه جمع آورى شد.

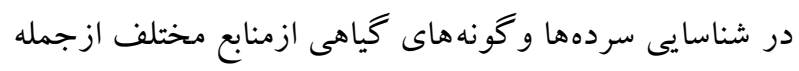

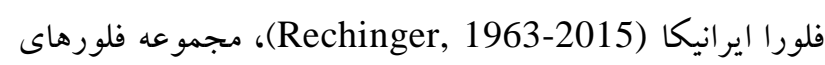

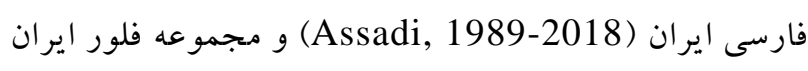

مolo

فلور، هر منطقه درحقيقت نتيجه واكنشهاى جامعه زيستى

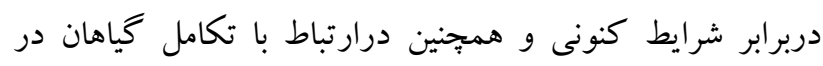

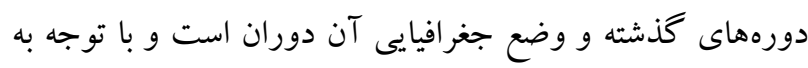

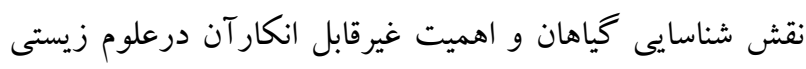

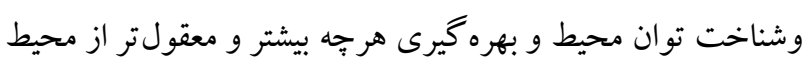

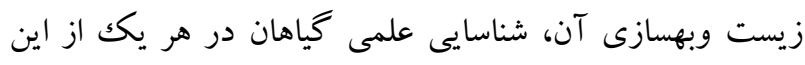

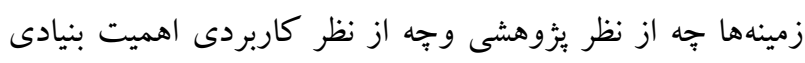

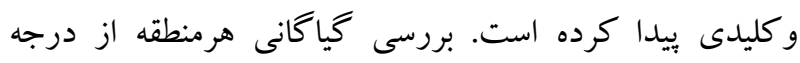

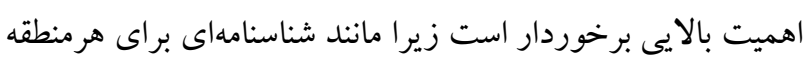

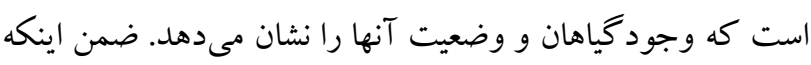

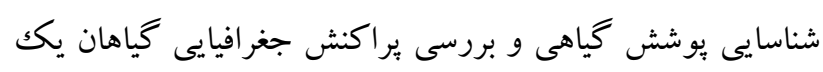

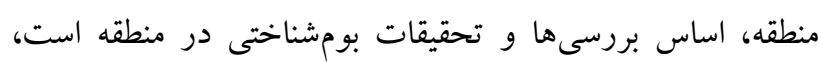

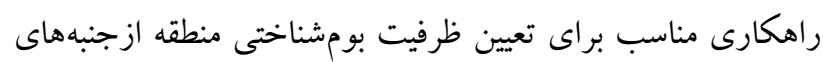

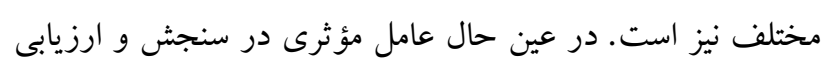

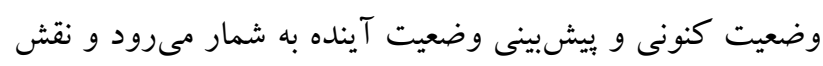
به بزائى براى اعمال مديريت صحيح درمنطقه دارد (

مطالعات يايه منطقه مورد مطالعه

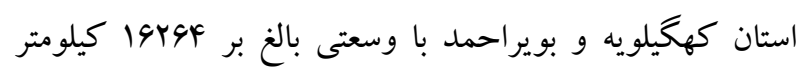

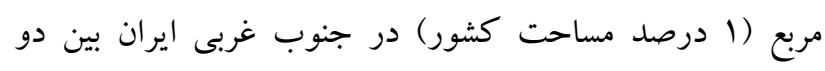
مدار 'F

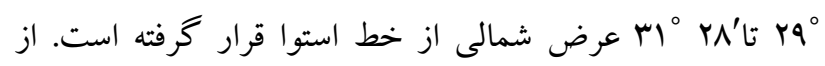

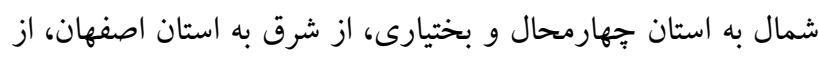
جنوب به استان فارس و بوشهر و از غرب به استان خوزستان

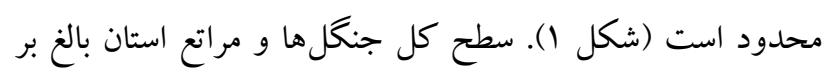
IF.....

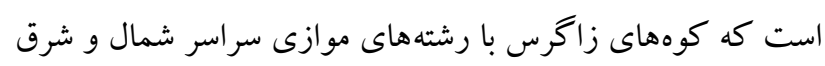

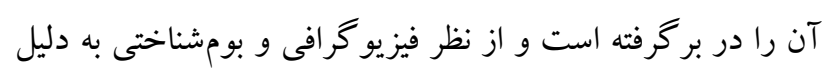

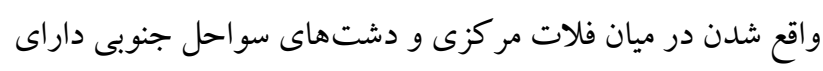

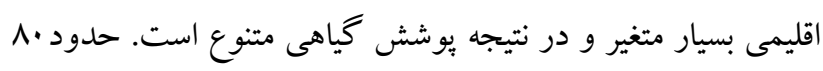

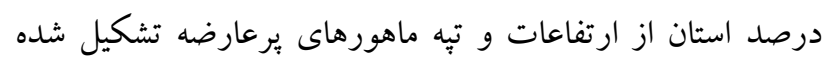

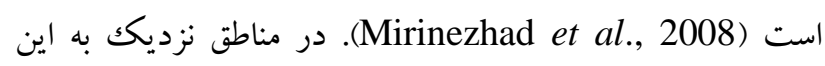
منطقه مطالعات مشابهى صورت گرفته است براى نمونه در بررسى

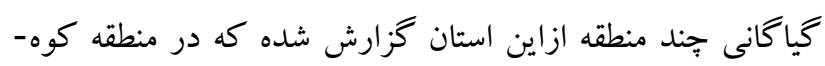




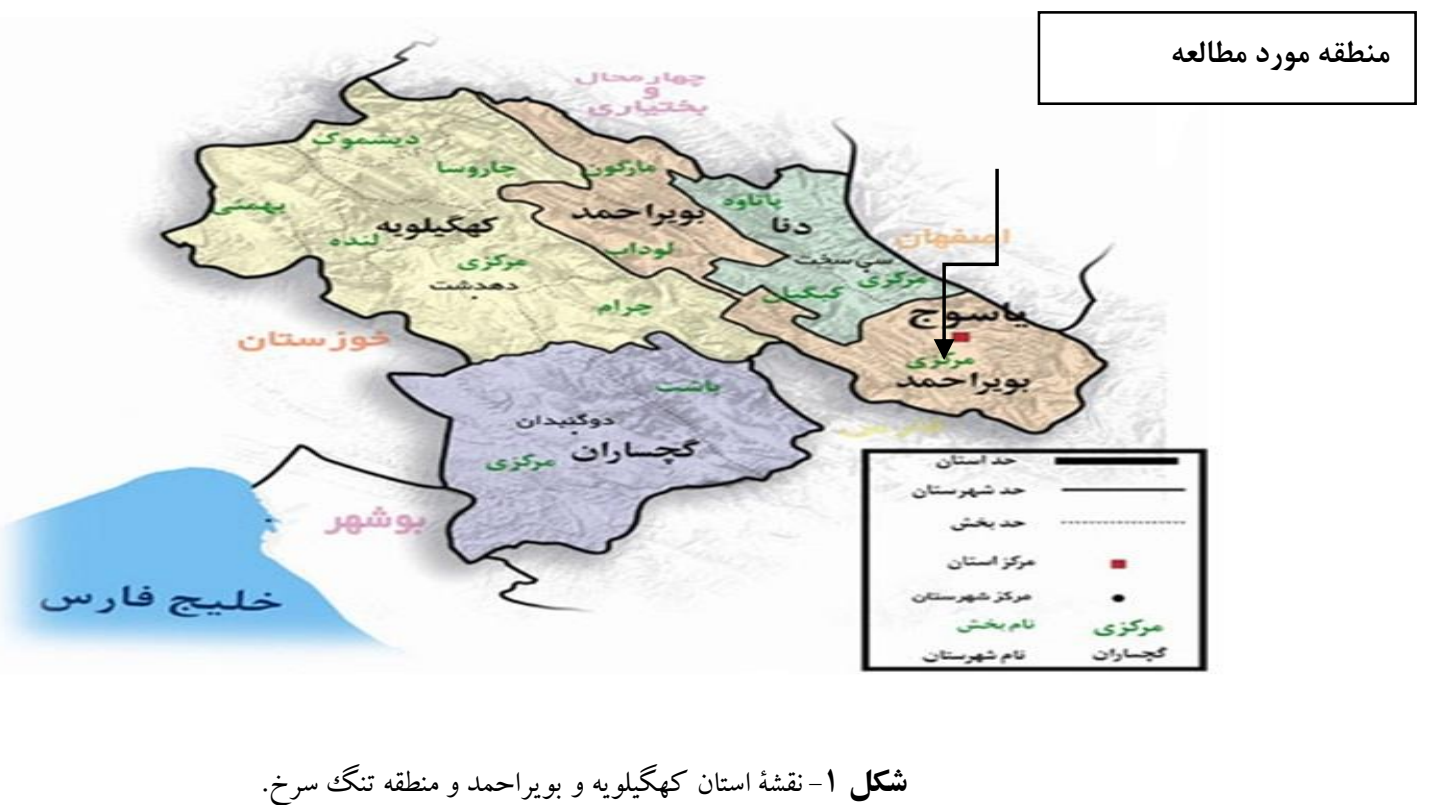

Fig. 1. Map of Kohgiloye va boyer- ahmad province and Tangsorkh region.

ترتيب عبارتند از تيره گندميان (Poaceae) با با گونه، وتيرهُ

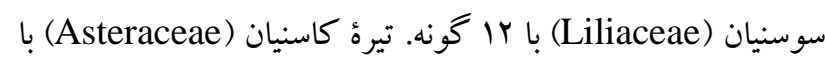

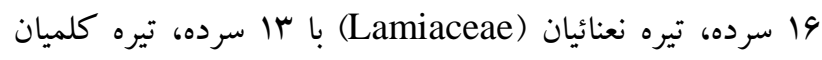
(Fabaceae) با با Y إده، تيره باقلائيان (Brassicaceae)

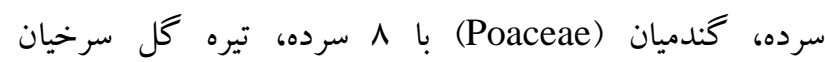

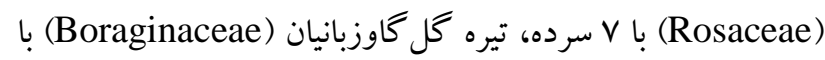

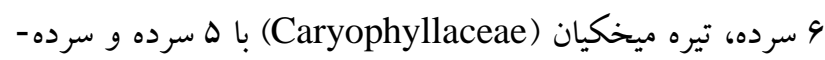

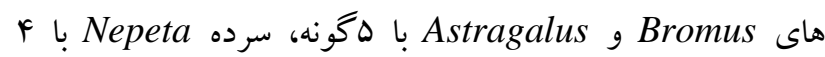

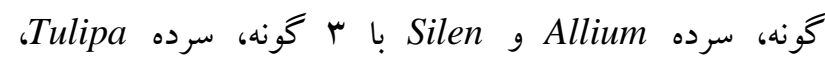
با Poa Salvia LLamium ،Fritillaria ،Ornithogolum

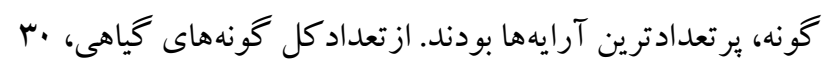

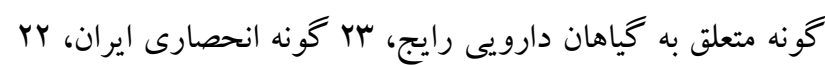

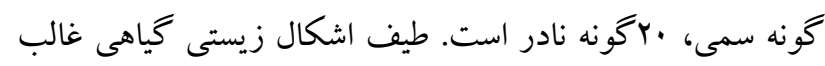

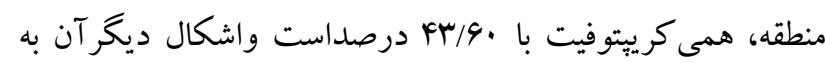

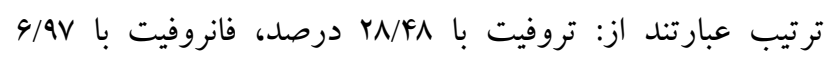

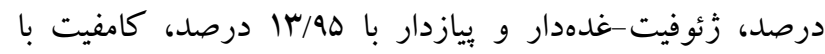

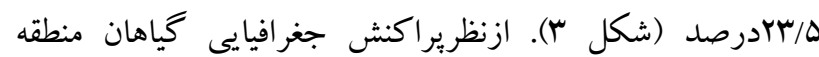

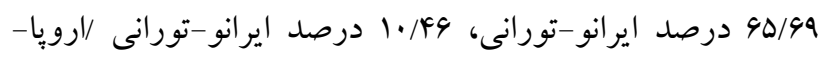

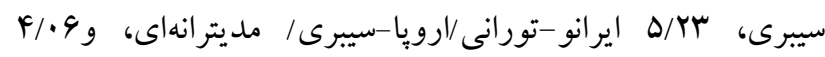

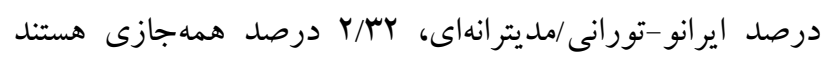

Maassoumi, ) (Ghahreman, 1978-2008)، گونهاى ايران 1986-2000) استفاده شده است. ضمن شناسايى گونه، فرم اشكال

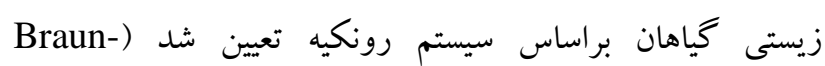

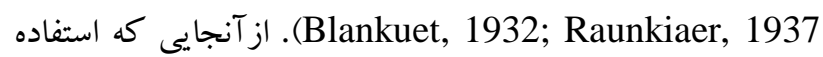
ازگياهان به خصوص گياهان دارويى درحال حاضر بسيار

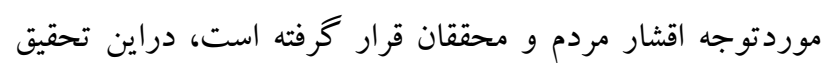

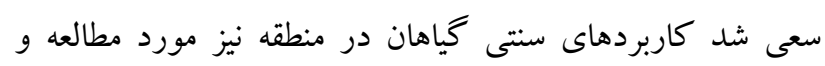

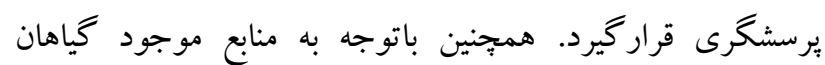

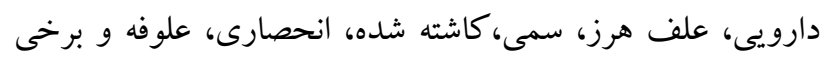
كاربردهاى معمول آنها بررسى شد.

\section{نتنإِج}

پس از بررسى بوشش گياهى اين منطقه فهرست كاملى از گياهان

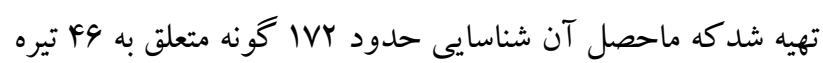

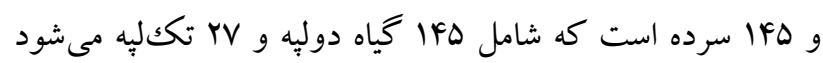
(جدول ضميمه (). بزر گترين تيرههاى دولِهاى به ترتيب عبارتند

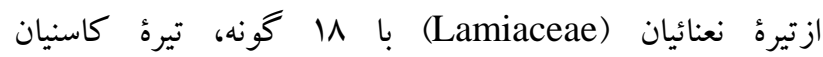

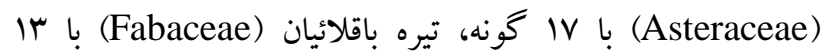
كونه، تيره كلميان (Brassicaceae) با سا گ گونه، تيره كرفسيان

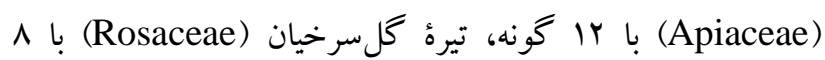

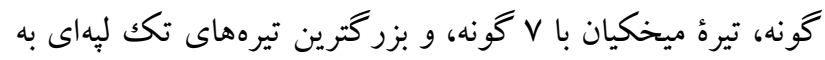




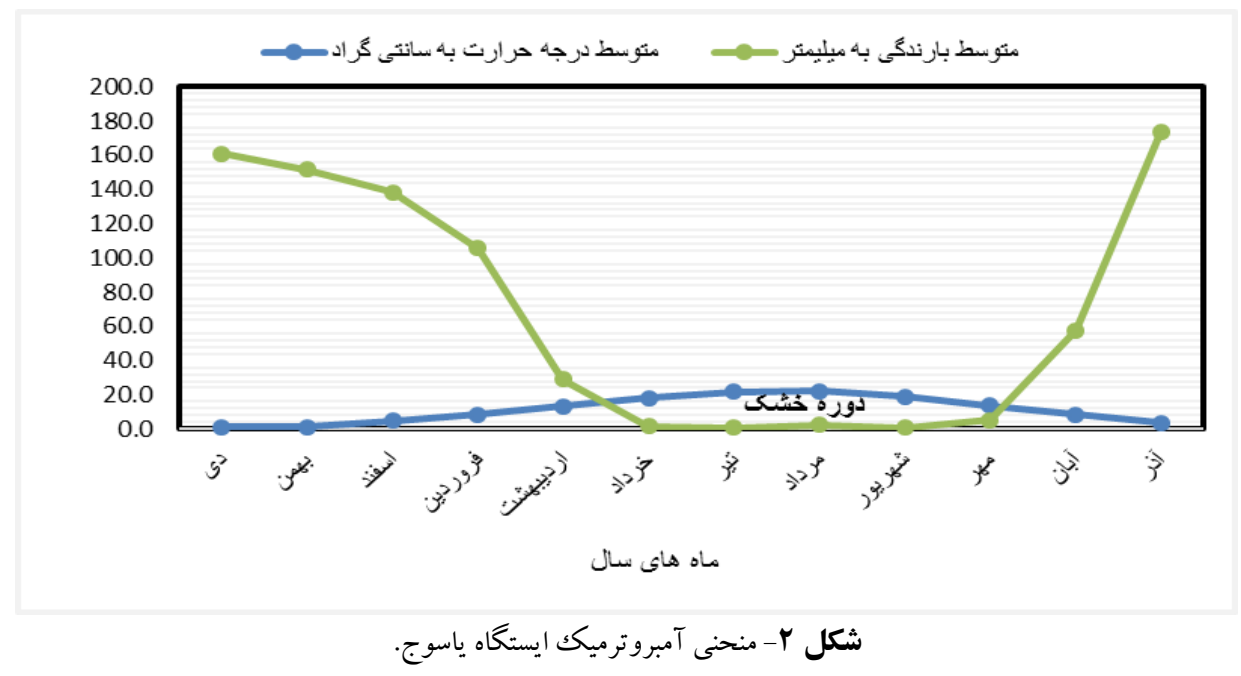

Fig. 2. Amberothermic curve of Yasuj station.

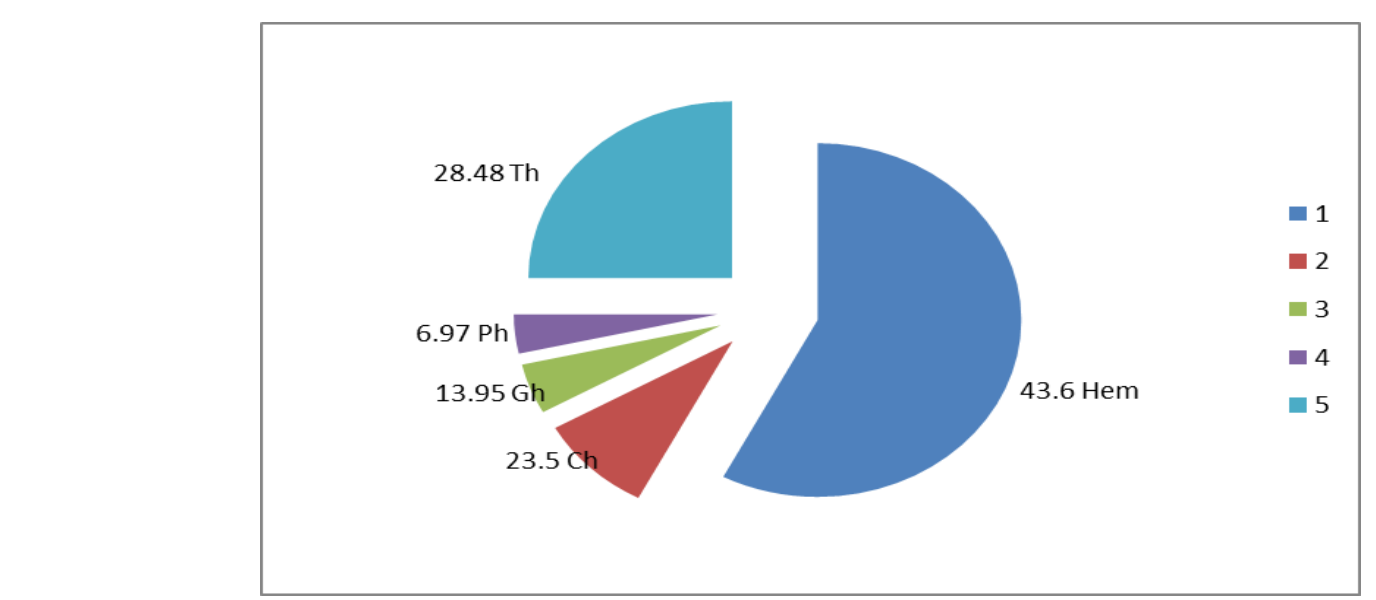

شكل r- نمودار فراوانى شكل هاى زيستى موجود در منطقه تنگك سرخ. Ch: كامفيت، Hem: همى كرييتوفيت، Gh: زئوفيت، Th: تروفيت، Ph: فانروفيت.

Fig. 3. The pie chart of species life form percentage in Tangsorkh region. Ch: Chamophyte, Hem: Hemichryptophyte, Gh: Geophyte: Th: Therophyte, Ph: Phanerophyte.

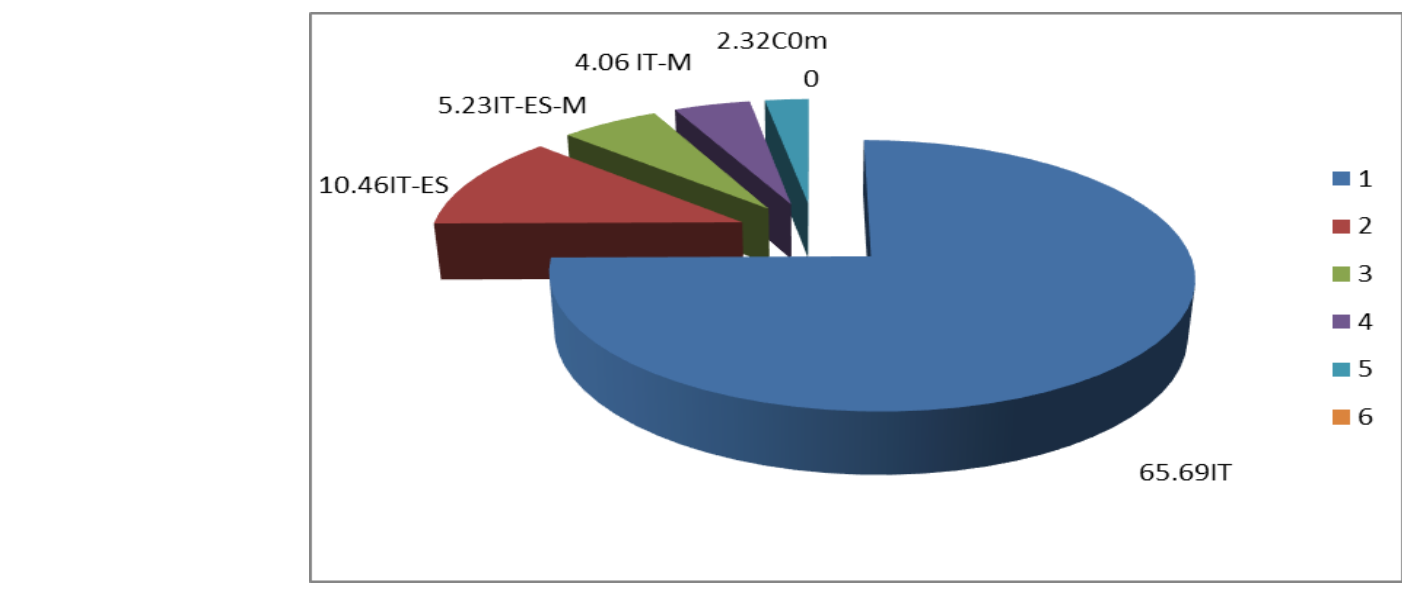

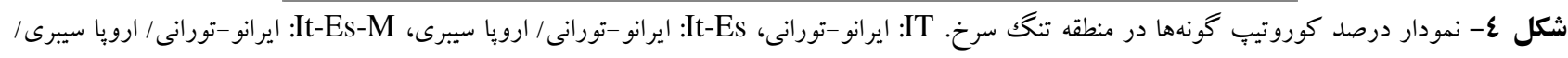

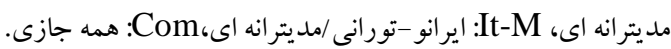

Fig. 4. The pie chart of percentage of phytochorya in Tangsorkh region. Ir.Tur: Irano-Turanian, IT-Es: Irano-Turanian/ European-Siberian, It-Es-M: Irano-Turanian/ European-Siberian / Mediteranean, It-M: Irano-Turanian / Mediteranean, , Cosm: Cosmopolitan. 


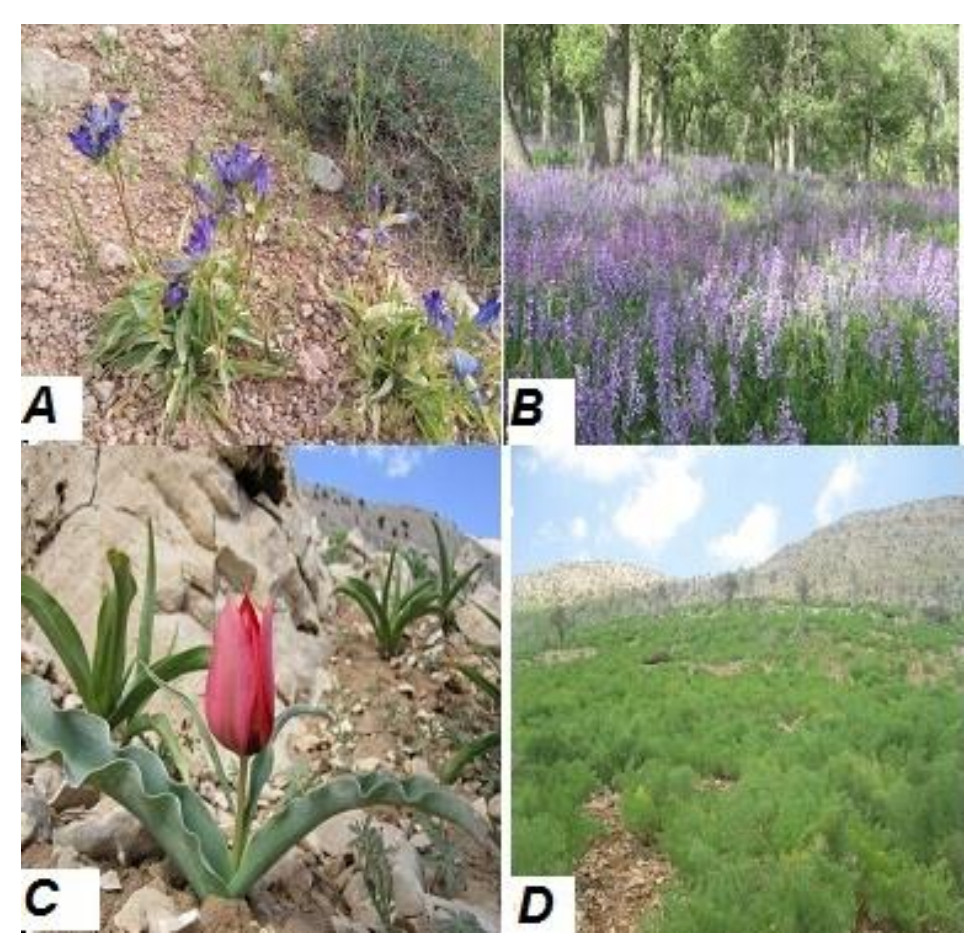

شكل ه- تصاويرى از برخى گياهان منطقه: Prangos ferulacea :D Fig. 5. Some images of plants of the region: A: Gentiana olivieri; B: Vicia ervilia; C: Tulipa montana; D: Prangos ferulacea.

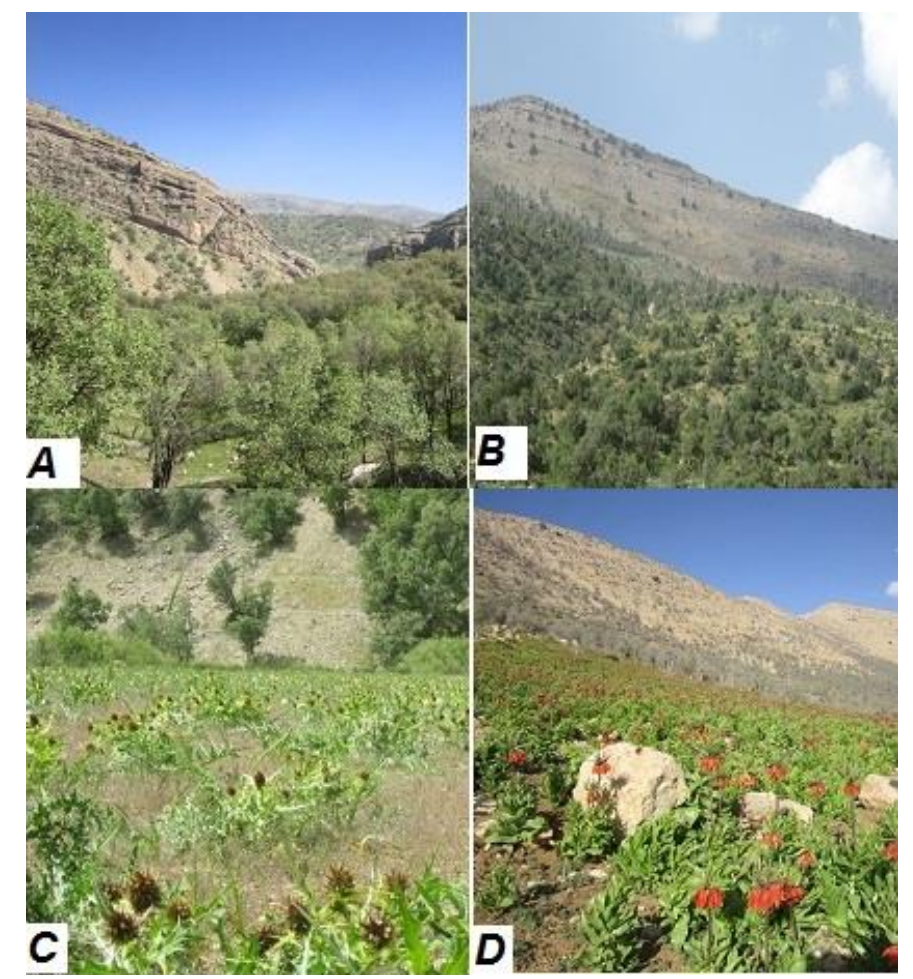

شكل 7- تصاويرى از برخى گياهان منطقه. Auercus brantii :A Fritillaria imperialis

Fig. 6. Some images of plants of the region: A: Quercus brantii; B: Amygdalus haussknechtii; C: Gundelia tournefortii; D: Fritillaria imperialis. 
منطقه مى موان به گياه آوندول ( Smyrnium cordifolium (Boiss. آن به صورت تازه و يا تنورى شده استفاده مى كنند.

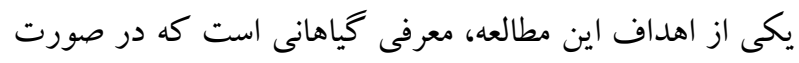

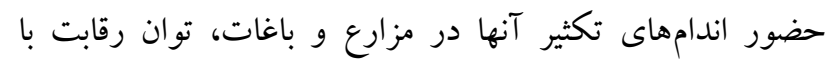

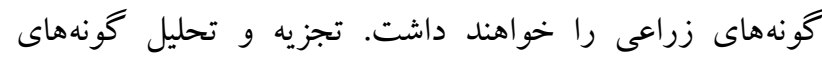

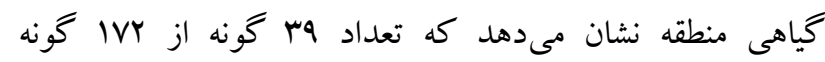
كياهى(Y/9V

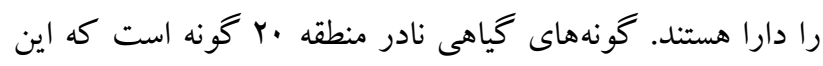

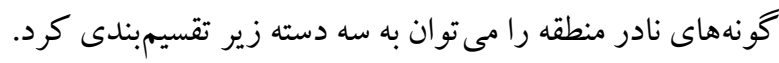

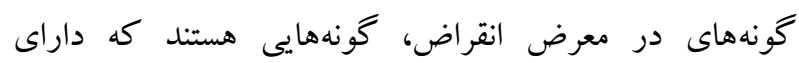

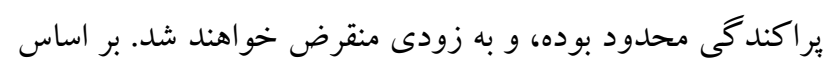

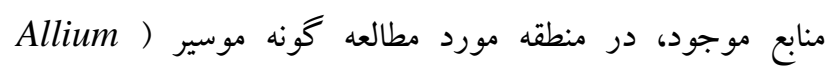
(hirtifolium Boiss.

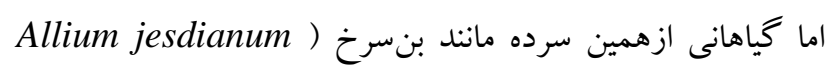
(Boiss. \& Buhse

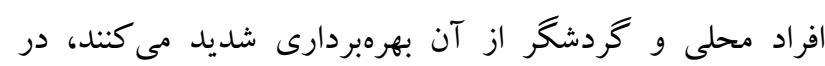

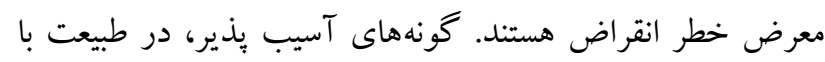
ريسك بالايى روبرو بوده و در صورت عدم حفاظت، در آينده

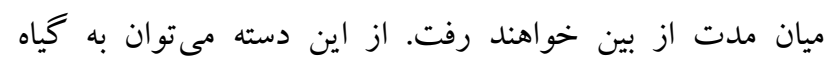

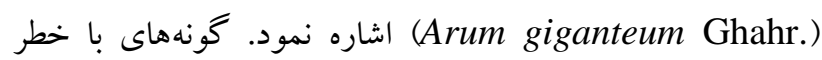

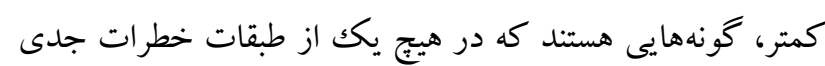

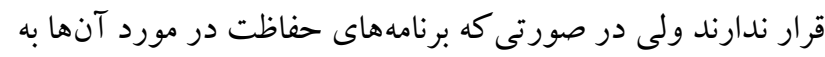

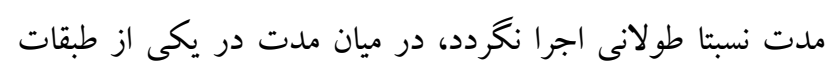

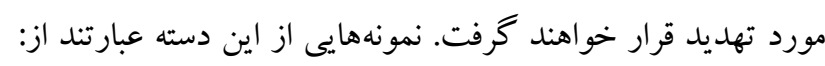

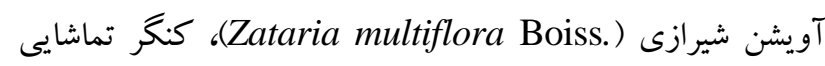
(Cirsium spectabile DC.)

ارزش حفاظتى در منطقه مورد بحث جزو اين طبقه قرار مى كيرند.

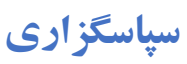

مراتب قدردانى خود را از مسئولان و همكاران مؤسسه منابع طبيعى، مؤسسه تحقيقات جنگلها و مراتع وكارشناسان اداره

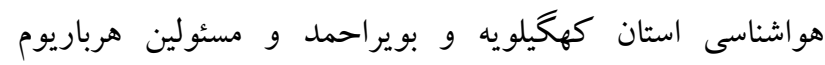
دانشكاه الزهراء كه در انجام اين تحقيق ما را يارى كردند اعلام
باتوجه به اينكه جراى دام باعث تخريب بوشش گياهى، كاهش

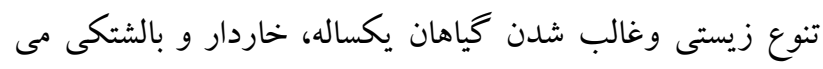

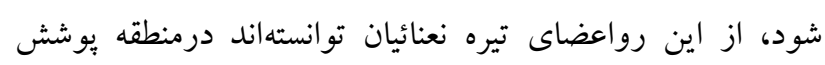

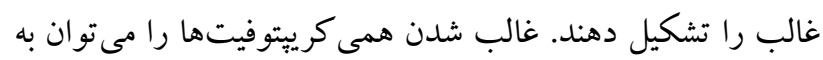
اقليم منطقه نسبت داد. باتوجه به اينكه اقليم منطقه سرد و كوهستانى است، لذا غالب شدن فرم زيستى همى كريتوفيت، نشاندهنده نيته

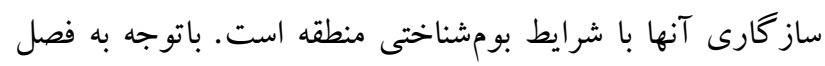

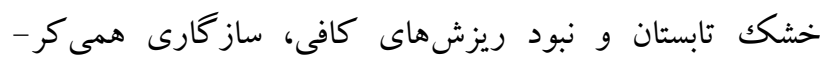

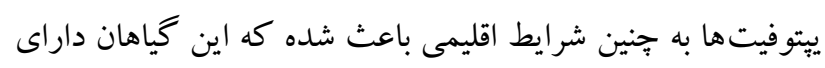

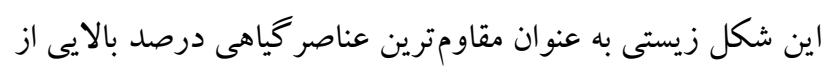

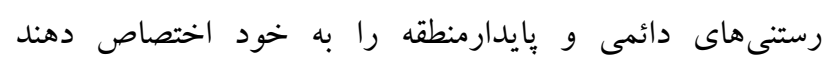
(Mobayen, 1982) مطالعات دقيق جغرافياى گياهى و زئوبو تانيكى تعيين مى گردد و و

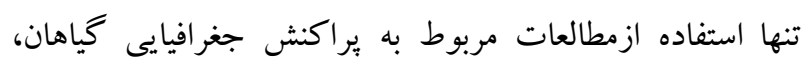

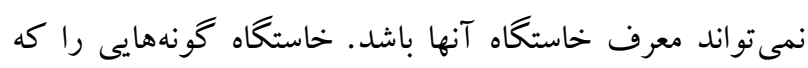
دريكك ناحيه رويشى ير اكنش دارند، مى توان با اطمينان بالا همان

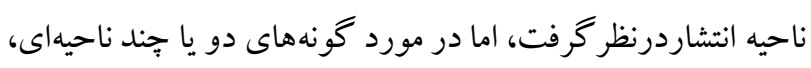
انتشار وسيعتر دريك ناحيه رويشى نمى تواند بيانكر خاستخاه آنها باشد. نتيجه مطالعات گونه هاى انحصارى نشان مىدهد كه درمنطقئ

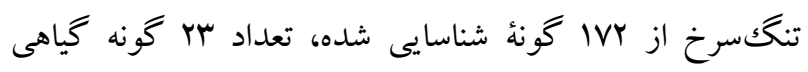

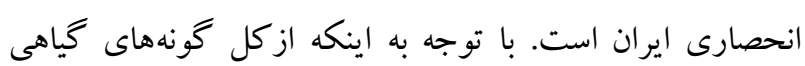

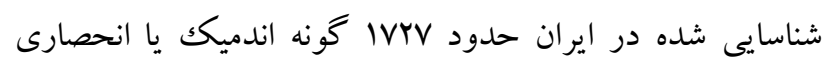

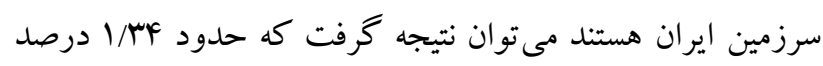

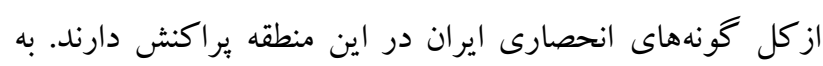

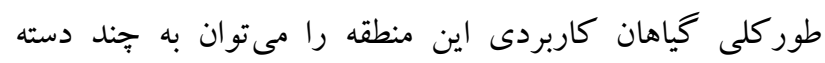
تقسيم كردكه شامل گياهان دارويى، سمى، نادر و گياهان هرز

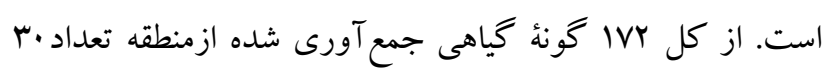

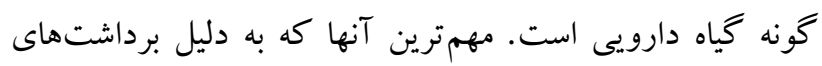

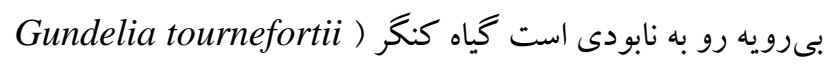

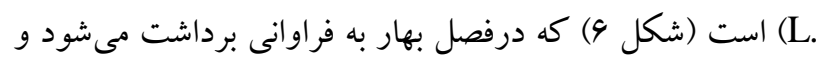
دربازارهاى سنتى ياسوج به فروش مىرسد. گياهان سمى كه تعداد

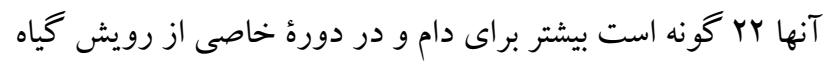

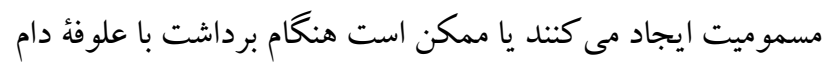

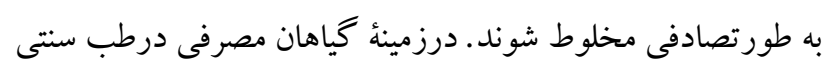




\section{REFERENCES}

Asri, Y. 1995. Plant ecology and vegetation. - PayameNoor Publication, Tehran. 209 pp.

Assadi, M. (ed.). 1989-2018. Flora of Iran, 1-149. RIFR, Tehran.

Braun-Blanquet, J. 1932. Plant sociology, The study of plant communities (Ttranslated by G.D. Fuller \& H.S. Conard, 1983). - McGraw Hill Book Company, Inc. New York, 439 p.

Ghahreman, A. 1978-2008. Flora of Iran. 1-26. - RIFR, Tehran.

Ghahreman, A. and Attar, F. 1999. Biological diversity of plant species. - Tehran University Press. 1176 pp.

Jalili, A. and Jamzad, Z. 1999. Red data book of Iran. Ministry of Jahad-e-Sazandegi. Tehran. 748 pp

Jani pour, R. 2015. Floristic study of mountains of North and North West of Choram Kohgiluyeh Boyerahmad province. Msc. thesis, Yasuj University, Yasuj.

Maassoumi, A. 1986-2000. Astragalus in Iran. Vols 1-4. - Research Institute of Forests and Rangelands, Tehran.

Mirinezhad, Sh. Youssefi, S. and Mozaffari, A. 2008. Biological diversity Study and ecology of plant species province of Kohgiluyeh \& Boyerahmad. - 1st International Symposium of Climate Change and Dendrochronology in Caspian Ecosystems. Sari Department of Natural Resources.

Mozaffarian, V. 1997. Iran culture plants (Latin, English and Persian). - Publication Contemporary Culture, Tehran.
Mozafarian, V. 2004. Plant classification. Vols. 1-2. Publication of Amirkabir, Tehran.

Raunkiaer, C. 1937. Plant life forms. - Oxford University Press, Clarendon, 162 pp.

Rechinger, K.H. (ed.). 1963-2015. Flora Iranica. 1-174: Akademische Druck- u. Verlagsanstalt, Graz; 175: Akademische Verlagsgesellschaft, Salzburg; 176-181: Naturhistorisches Museum, Wien.

Zohary, M. 1973. Gebotanical foundations of the Middle East. 2 vols. - Gustav Fischer Verlag, Stuttgart, Germany, $738 \mathrm{pp}$.

Zargari, A. 2012. Medicinal plants, vol. 1-5. - Tehran University Press. Tehran.

Zarifian, A. 2014. Sawers mountain floristic study in Kohgiluyeh va Boyerahmad. - Msc. thesis, Yasuj University. Yasuj.

How to cite this article:

Moradpoor, S., Pakravan, M. and Jafari, A.O. 2019. The Floristic study of Tang-sorkh region of Kohgiloyeh va Boyerahmad province. - Nova Biol. Reperta 2019: 403-410.

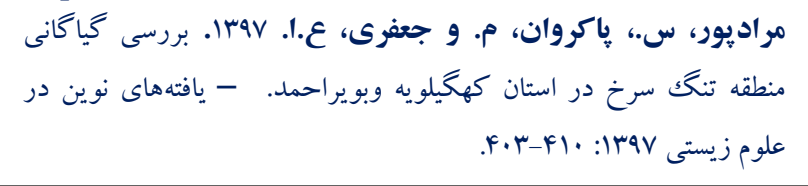

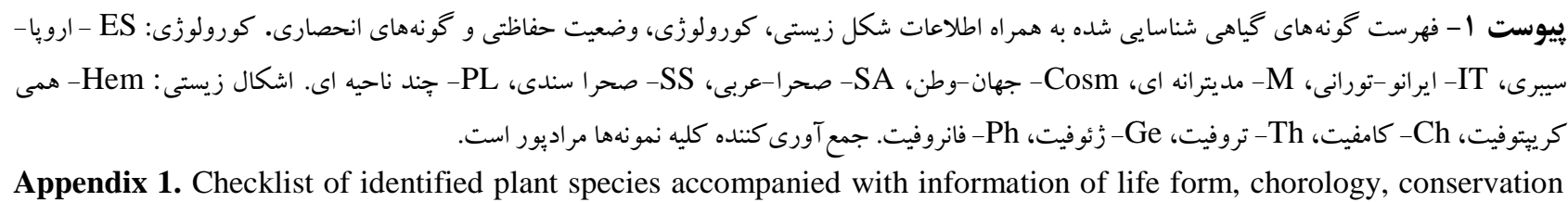
status and endemic species. Chorology: ES- Eurosberian, , IT- Irano-Turanian, M- Mediteranean, Cosm- Cosmopolitan, SA- Sahara-arabian, SS - Saharo-Sindian, PL- Polyregion. Life form: Hem- Hemichryptophyte, Ch- Champhyte, ThThrophyte, Ge- Geophyte, Ph- Phanerophyte. The total specimens are collected by Moradpoor.

Amaryllidaceae: Allium ampeloprasum L. subsp. iranicum Wendelbo [Ge; IT] (7023); Allium jesdianum Boiss. \& Buhse [Ge; IT] (7024); Allium haemanthoides Boiss. Reut. ex Regel [Ge; IT] (7025); Anacardiaceae: Pistacia atlantica Desf. [Ph; IT] (7002). Apiaceae: Bupleurum gerardii All. [Th; IT] (7151); Chaerophyllum macropodum Boiss. [Hem; IT] (7152); Dorema aucheri Boiss. [Hem; IT] (7153); Ferulago angulata (Schltdl.) Boiss. [Hem; IT] (7155); Ferula gummosa Boiss. [Hem; IT] (7156); Prangos ferulacea (L.) Lindl. [Hem; IT] (7158); Pimpinella puberula (DC.) Boiss. [Ge; IT] (7160); Scandix stellata Banks \& Sol. [Th; IT-ES-M] (7161); Smyrnium cordifolium Boiss. [Hem; IT] (7163); Torilis leptophylla (L.) Rechb.f. [Th; IT] (7165); Smyrniopsis aucheri Boiss. [Hem; IT] (7166); Foeniculum vulgare L. [Hem; IT] (7168). Araceae: Arum giganteum Ghahr. [Ge; IT] (7003); Asparagaceae: Bellevalia glauca (Lindl.) Kunth [Ge; IT] (7026). Muscari tenuiflorum Tausch [Ge; IT] (7030); Asteraceae: Achillea wilhelmsii K.Koch [Hem; IT-ES-Nb] (7004); Anthemis odontostephana Boiss. subsp. odontostephana [Th; IT] (7005); Centaurea behen L. [Hem; IT] (7006); Centaurea solstitialis L. [Hem; IT] (7007); Chardinia orientalis Desf. [Th; IT] (7008); Cirsium spectabile DC. [Hem; IT] (7009); Cousinia bachtiarica Boiss. \& Hausskn. [Hem; IT] (7010); Crepis sancta (L.) Bornm. [Th; IT-M] (7011); Crupina crupinastrum (Moris) Vis. [Th; IT-M] (7012); Echinops cyanocephalus Boiss. \& Hausskn. [Hem; IT] (7013); Eryngium billardieri Delile [Hem; IT] (7014); Gundelia tournefortii L. [Hem; ITM] (7015); Scariola orientalis (Boiss.) Sojak. [Hem; IT] (7016); Serratula bachtiarica Boiss. \& Hausskn. ex Boiss. [Hem; IT] (7017); Scorzonera sp. [Hem; IT] (7018); Tanacetum polycephalum Sch.Bip. subsp. polycephalum [Hem; IT-ES] (7019); Taraxacum sp. [Hem; -] (7020); Tragopogon buphthalmoides (DC.) Boiss. [Hem; IT] (7021); Boraginaceae: Arnebia euchroma (Royle) I.M.Johnst. [Hem; IT] (7046); Lappula barbata (M.Beib.) Gurke. [Hem; IT- 
M] (7047). Nonea pulla (L.) DC. [Hem; IT-ES] (7048). Brassicaceae: Hesperis kurdica F.Dvořák \& Hadač [Hem; IT] (7034); Chorispora tenella (Pall.) DC. [Th; IT] (7035); Aethionema carneum (Banks \& Sol.) B.Fedtsch. [Hem; IT] (7036); Alyssum dasycarpum Stephan ex Willd. [Th; IT-ES] (7037); Alyssum heterotrichum Boiss. [Th; IT] (7038); Cardaria draba (L.) Desv. [Th; cosm] (7039); Conringia persica Boiss. [Th; IT] (7040); Descurainia sophia (L.) Webb ex Prantl [Th; IT-ES-M] (7041); Erysimum repandum L. [Th; IT] (7042); Fibigia macrocarpa Boiss. [Hem; IT] (7043); Matthiola sp. [Th; IT] (7045). Caprifoliaceae: Lonicera nummularrifolia Jaub \& Spach. [Ph; IT] (7049); Pterocephalus canus Coult. ex DC. [Hem; IT] (7060); Scabiosa persica Boiss. [Th; IT] (7061); Valerianella discoidea (L.) Loisel. [Th; IT] (7170); Valerianella tuberculata Boiss. [Th; IT] (7171). Caryophyllaceae: Cerastium dichotomum L. [Th; Plur] (7051); Gypsophila sp. [Th; -] (7052); Holosteum glutinosum (M.Bieb.) Fisch. \& C.A.Mey. [Th; IT] (7053); Silene albescens Boiss. [Hem; IT] (7054); Silene chlorifolia SM. [Hem; IT] (7055); Silene chaetodonta Boiss. [Hem; IT] (7056); Vaccaria grandiflora Jaub. \& Spach [Th; IT] (7057). Colchicaceae: Colchicum autumnale L. [Ge; IT] (7059). Convolvulaceae: Convolvulus arvensis L. [Hem; IT-ES-Nb] (7022). Cyperaceae: Carex divisa Huds. [Ge; IT-ES] (7050). Euphorbiaceae: Euphorbia chiradenia Bioss. [Hem; IT] (7062); Euphorbia macrostegia Boiss. [Ge; IT] (7063). Elaegnaceae: Elaeagnus angustiofolia L. [Th; IT] (7064). Fagaceae: Quercus Brantii Lindl. [Ph; IT] (7065). Fabaceae: Astragalus cephalanthus DC. [Ch; IT](7099); Astragalus rhodosemius Boiss. \& Hausskn. [Ch; IT] (7100); Astragalus ovinus Boiss. [Hem; IT] (7101); Astragalus sp. [Hem; IT] (7102); Astragalus chalaranthus Boiss. \& Hausskn. [Hem; IT] (7103); Lotus tenuis Waldst. \& Kit. [Hem; IT] (7104); Medicago sativa L. [Hem; cosm] (7105); Onobrychis cornuta (L.) Desv. subsp. cornuta [Ch; IT] (7106); Oxytropis sp. [Hem; -] (7107); Trifolium pratense L. [Th; IT] (7108); Vicia ervilia (L.) Willd. [Th; IT-ES] (7109); Sophora alopecuroides L. [Hem; IT] (7110); Glycyrrhiza glabra L. [Ge; IT-ES] (7111). Gentianaceae: Gentiana olivieri Griseb. [Hem; IT-M] (7067). Geraniaceae: Erodium cicutarium (L.) L' Hẻr [Th; IT-ES-M] (7068); Geranium tuberosume L. [Ge; IT] (7069); Biebersteinia multifida DC. [Ge; IT] (7070). Hypericaceae: Hypericum scabrum L. [Hem; IT] (7071). Iridaceae: Gladiolus italicus Mill. [Ge; ITES-NB] (7072). Ixioliriaceae: Ixiolirion tataricum (Pall.) Schult. \& Schult.f. subsp. montanum (Labill.) Takht. [Ge; IT] (7073). Juncaceae: Juncus inflexus L. [Hem; cosm] (7074). Lamiaceae: Acinos graveolens Link. [Th; IT] (7075); Ajuga austroiranica Rech.f. [Hem; IT] (7076); Ballota aucheri Boiss. [Hem; IT- SS] (7077); Eremostachys laevigata Bunge. [Hem; IT-M](7078); Lamium album L. subsp. crinitum (Montbr. \& Auch. ex Benth.) Mennema [ES; IT] (7079); Lamium perspolitanum (Boiss.) Jamzad [TH; IT] (7080); Marrubium vulgare L. [Hem; IT-ES] (7081); Mentha longifolia (L.) L. [Hem; IT] (7082); Nepeta daenensis Boiss. [Th; IT] (7083); Nepeta kotschyi Boiss. subsp. kotschyi [Hem; IT] (7084); Nepeta schiraziana Boiss. [Th; IT] (7085); Nepeta macrosiphon Boiss. [Hem; IT] (7086); Phlomis oliveri Benth. [Hem; IT-ES] (7087); Salvia persepolitana Boiss. [Hem; IT-Nb] (7088); Salvia sclarea L. [Hem; IT-ES] (7089); Satureja bakhtiarica Bunge. [Hem; IT] (7090); Stachys pilifera Benth. [Hem; IT] (7091); Zataria multiflora Boiss. [Hem; IT-Nb] (7092). Liliaceae: Fritillaria imperialis L. [Ge; IT] (7027); Fritillaria persica L. [Ge; IT-Sh.a] (7028); Gagea gageoides (Zucc.) Vved. [Ge; IT] (7029); Ornithogalum umbellatum L. [Ge; IT] (7031); Tulipa biflora Pall. [Ge; IT-ES] (7032); Tulipa montana Lindl. subsp. montana [Ge; IT] (7033). Malvaceae: Malva neglecta Wallr. [Hem; IT-ES-M] (7094); Alcea Kurdica L. [Hem; IT] (7095). Oleaceae: Fraxinus rotundifolia Miller subsp. persica (Boiss.) A.E.Murray [Ph; IT] (7096). Orobanchaceae: Orobanche sp. [Ge; -] (7097). Papaveraceae: Papaver dubium L. [Hem; IT] (7098); Fumaria parviflora Lam. [Th; Plur] (7066). Plantaginaceae: Plantago lanceolata L. [Hem; ITES-Nb] (7112); Veronica orientalis Miller. [Hem; IT-M] (7148). Plumbaginaceae: Acantholimon flexuosum Boiss. ex Bunge [Ch; IT] (7113). Poaceae: Aegilops triuncialis L. [Th; IT] (7114); Alopecurus apiatus Ovcz. [Hem; IT] (7115); Bromus danthoniae Trin. ex C.A.Mey. [Th; IT-ES] (7116); Bromus scoparius L. [Th; IT-ES-M] (7117); Bromus sterilis L. [Th; IT-ES] (7118); Bromus tectorum L. subsp. hirsutus Regel [Th; cosm] (7119); Bromus sericeus Ten [Th; IT] (7120); Hordeum bulbosum L. [Hem; IT-ES-M] (7121); Heteranthelium piliferum Hochst. ex Jaub. \& Spach [Th; ITES-M] (7122); Poa pratensis L. [Hem; plur] (7123); Poa bulbosa L. [Ge; IT] (7124); Stipa barbata Desf. [Th; IT] (7125). Podophyllaceae: Bongardia chrysogonum (L.) Spach. [Ge; IT-ES-Nb] (7126). Ranunculaceae: Adonis aestivalis L. [Th; IT-ES-M] (7127); Anemone biflora DC. [Hem; IT] (7128); Ceratocephalus falcatus (L.) Pers. [Th; ITES] (7129); Ranunculus grandiflorus L. [Hem; IT] (7130); Thalictrum isopyroides C.A.Mey. [Hem; IT-ES-M] (7131). Rosaceae: Amygdalus haussknechtii C.K.Schneid ex Bornm. [Ph; IT] (7132); Cerasus microcarpa (C.A.Mey.) Boiss. subsp. microcarpa [Ch; IT] (7133). Cerasus pseudoprostrata Pojark. [Ch; IT] (7134); Cerasus mahlab (L.) Mill. [Ch; IT] (7135); Crataegus pontica K.Koch. [Ch; IT] (7136); Rosa canina L. [Ch; IT] (7137); Rubus sanctus Schreber [Ph; IT-ES] (7138); Sanguisorba minor Scop. subsp. muricata (Spach.) Briq. [Hem; IT-ES-M] (7139); Pyrus glabar Boiss. [Th; IT] (7140). Rubiaceae: Callipeltis cucullaria (L.) DC. [Th; IT] (7141); Cruciata taurica (Pall. ex Willd.) Ehrend. [Th; IT] (7142); Galium aparine L. [Th; IT] (7143). Salicaceae: Populus alba L. [Ph; IT] (7144); Salix acmophylla Boiss. [Ph; IT] (7145). Santalaceae: Viscum album L. [par; IT-ES-Sh.a] (7093). Sapindaceae: Acer monspessulanum L. subsp. persicum (Pojark.) Rech.f. [Ph; IT] (7001). Scrophulariaceae: Scrophularia nervosa Benth. subsp. boissierana (Jaub. \& Spach) Grau [Hem; IT] (7146); Scrophularia striata Boiss. [Hem; IT] (7147). Thymelaeceae: Daphne mucronata Royle. [Ph; IT] (7149). Tamaricaceae: Tamarix ramosissima Ledeb. [Ph; IT] (7150). Violaceae: Viola odorata L. [Th; IT-ES] (7172). 